\title{
Fusion Energy: On the track of a different philosophy and solution
}

\begin{abstract}
When two (or more) nuclei fuse, to form a heavier element, energy is released. The mechanism is known, at least to some degree. And the actual technological development on the subject has been introducing applications in many areas. Even so, there are some challenges before we can be able to provide energy by an appropriated fusion process: high needs of power, plasma confinement issues, heavy equipment, etc.

The present idea intends to show a different view. The author calls in a broad sense, Conceptual Thesis, and it has the Natural Language as principal tool. The author either explicitly or implicitly discusses the space-time fabric, double-slit experiment, and other concepts like non-duality. A nano-apparatus draft with the purpose to be in the scope of a near future research, closes the picture.
\end{abstract}

Keywords: Fusion Energy, Nanodevice, Conceptual Thesis.

\section{Introduction}

Fusion with the production of a large amount of energy is a technology that can be used in a very positive way by our society and be a factor to contribute to the natural balance of the environment.

This study aims to present a possible compromising answer about a different physical-theoretical system. The model is supported by a conceptual thesis/thought, precursor of a probable widereaching symbolic-informative relational theory.

The idea under test is that atomic particles can be fused not by a process where the Coulomb barrier is overcome, but the particles, under certain conditions, or rather, by altering and/or maintaining certain properties (connectedness), can break the space-time fabric, find themselves at an undefined point (non-dual and/or suspended from their space-time frame), and finally return to the real state, whose paradigm rests on a distinction of the particles properties, with the release of energy.

General process. It is considered one antagonistic property of the particles to be resolved. If the environment is, say, equivalent, and the particles are put in a right configuration apparatus, then one opposite property remains and the particles are able to start the fusion process. (One antagonistic property is to say two antagonistic possible measurements, yes and no - to produce Non-duality in a conceptual approach.) After non-duality happens and after the return to the three-dimensional world frame, the particles are approximately one, i.e., they are, by principle, together (bonded). 
Process: rupture of the space-time fabric. The particles are disconnected from the space-time fabric of numerical quality. (Space-time is considered to be intrinsically of numerical quality.)

As particles start to become identified as one, means a rupture of space-time: the numbers of their initial frame tend to be equal conceptually - concept of independent number ceases to exist.

Process: Non-duality. An element exists with properties or response that approximates and eliminate the antagonisms: it induces in the system a conceptual change. This conceptual change is viewed as the loss of differentiation of the particles - particles are identified with a single property (in this case study, distance - Fig. 1). What the author calls weak and strong reference (Fig. 4), are fundamental for this change to happens.

Applying the apparatus system (which will permit such all process) by setting it at initial conditions, the particle(s) return to the three-dimensional world frame.

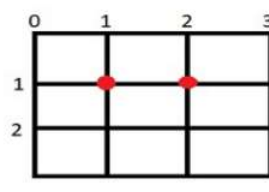

(1)

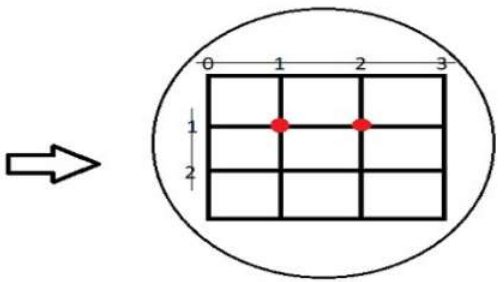

(2)

Figure 1. Change on the reference of the particles.

(1) Two similar particles with reference: the property position is known;

(2) Two similar particles without reference: the property distance is the same (by definition).

\section{Objective}

The objective of this work is to give a new approach on the fusion process with the aim of further research to take place in the labs, for that:

- The paper introduces some philosophical concepts into the physics of fusion.

- A first draft of a nanodevice for the produce of energy by the fusion process is presented.

\section{Method}

Apart of what was said above, the qualitative approach can be resumed by the following.

The double-slit experiment was revisited considering a role for information. It results a simple scheme shown on figure 2 and 3. Already accordingly (but not aware) with that view, space-time was resolved as two dimensions plus time, in a conceptual understanding or description of it. With this view of space-time fabric, the idea of a particle losing its time by suppressing its Z-Axis, starts the journey to design the device. The device accomplishes the points written for the process. A representation of it and some conceptual notions of the process can be viewed on Thought Results. 


\section{Thought Results}

The double-slit experiment interpreted by the particle behaviour of particles with mass. The informational line has constant speed. (Particle's maximum kinetic energy before a conceptual change occurs?)

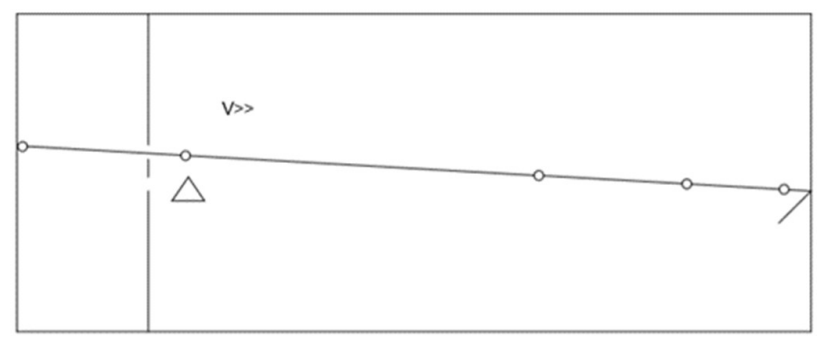

Figure 2. Double-slit thought experiment (view from top): the particle (an atom) gain momentum transmitted by the detector and its velocity increases.

Below, one can observe a particle, changing from the probably most intuitive path to a not so intuitive one, at the node and due to its lower initial velocity.

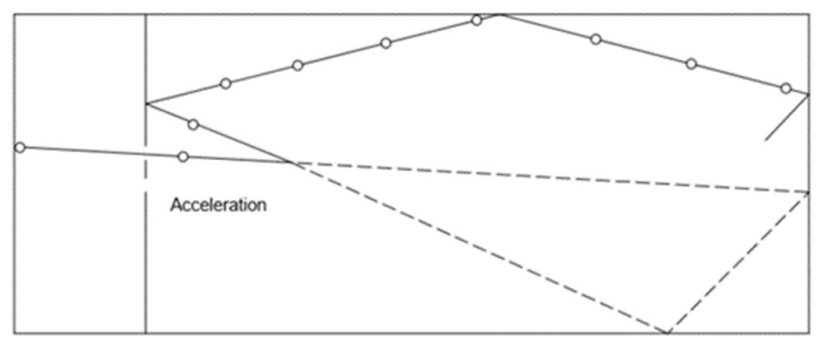

Figure 3. Double-slit thought experiment (view from top): the particle (an atom) moving slower than its informational line, is pulled to the node where the line crosses - acceleration; time updated at node; the particle does not take the dashed path.

Space-time fabric with three dimensions: space with two dimensions, plus time, in a pure conceptual approach. Z-Axis and time are identical concepts considering that one can figure out the property position, having knowledge of initial velocity, time, and the two-dimensional path. If space-time is suppressed, connectedness between particles changes. This is achieved by return to 0 on Z-Axis of the nanodevice, where also initial conditions are setting for the particles.

Nanodevice draft. A simple scheme of a device: the device carries particles and two references are built in: a Weak Reference (WR), a Strong Reference (SR). The apparatus process starts with the top of the coupled parts in position P1. SR and WR switched on. As soon movement 1 happens SR decreases. At position P2, SR switches off and movement 2 takes place (Ring and WR going together) with WR intermittent. When Ring arrives to position P1, WR switches off and moves in the opposite way (movement 3) until stops at P1. The SR and WR are switched on. 
The atoms of type A are differentiated by the intervention of the type A ions, meaning the electrons apart of the nucleus. Is now considered that the atoms do not need to be ionized.

The energy can be controlled by the quantity of atoms to be used in the process.

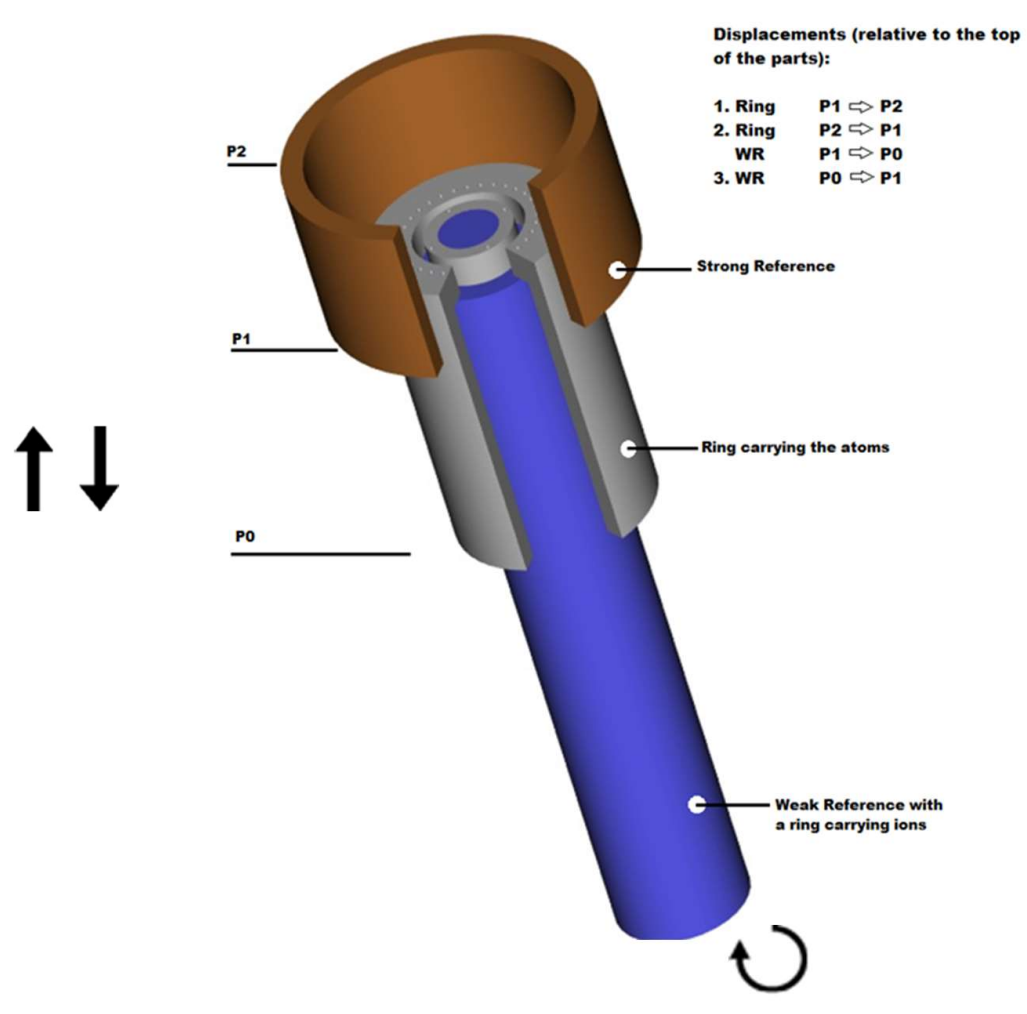

Figure 4. Draft of a nanodevice for fusion energy.

\section{Discussion}

The double-slit experiment is an exercise for the propose of building the nanodevice. No experiments (by simulation or real) were done. The thought experiments are well reasoned. The main doubt is if the expected results are in accordance with the well distributed wave function results.

The device is novel in theory, and itself represents a possible shift in the understanding of some important concepts. There is no reference to the materials to be used (its design only must be observed after other positive conclusions of this study). Making use of the latest technology, is possible to achieve a good result.

The proposal presented must be subject of further research, and experimental results are of extreme importance.

\section{Conclusions}

A coherent model of a fusion energy device and all considered different theories, seems to work conceptually. Some of them are not fully developed.

The language used, lacks formal aspects, but was the mean to design the device.

In the author opinion, is an advantage to present this article at this stage, once one can prove or disprove the contents, and contribute to a better understanding of Fusion and Nature. 


\section{Acknowledgements}

I due my thanks to all my professors and other teachers that I met along this formation journey.

I am grateful to my family and friends for their support in my life.

\section{Author Contributions}

João Pedro Ferro is the single author and there are not external contributions.

\section{Funding information}

Due to the characteristics of the work that has been done, no list of financial support exists. It is worth to stress that is an individual, theoretical study, with the objective of pursuit its development in laboratory.

\section{Data availability}

Data availability is not applicable to this article as no tangible data were created or analysed in this study.

\section{References [note]}

References are not applicable to this study as it is entirely original. Only general previous reading was made.

To notice that $2+1$ space-time fabric has been worked in the study of black holes and other cosmological studies; there exist some notions of fabric rupture, but not systematised in order to become a theory. 